\title{
Anatomía del culmo de bambú (Guadua aculeata Rupr.) de la región nororiental del estado de Puebla, México
}

\section{Bamboo culms anatomy (Guadua aculeata Rupr.) of the north-east region of Puebla state, Mexico}

\author{
Idalia Zaragoza-Hernández', Amparo Borja de la Rosa', Francisco José Zamudio Sánchez', \\ Víctor Rubén Ordóñez-Candelaria² y Guadalupe Martha Bárcenas-Pazos²
}

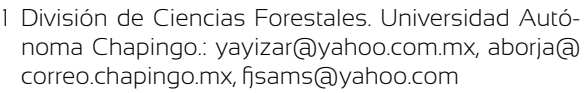

I División de Ciencias Forestales. Universidad Autónoma Chapingo.: yayizar@yahoo.com.mx, aborja@a correo.chapingo.mx, fisams@yahoo.com

2 Red de Ambiente y Sustentabilidad. Instituto de Ecología, A.C. victor.ordonez@inecol.mx, guadalupe. barcenas@inecol.mx

\section{RESUMEN}

Se realizó la caracterización anatómica de la pared de los culmos de Guadua aculeata Rupr., los especímenes fueron colectados en el predio "Las Margaritas" ubicado en el municipio de Hueytamalco, Puebla, México. El objetivo fue conocer la proporción de las estructuras anatómicas que componen el culmo y las dimensiones de las fibras, para contribuir al conocimiento de la especie. Se estudió la composición anatómica del culmo y se hizo la caracterización estructural de las fibras; en ambos casos se utilizaron cuatro culmos. Para la medición de las fibras, cada culmo se seccionó en tres partes de su longitud (inferior, medio y superior), de estas secciones se cortaron rodajas del entrenudo y cercanas al nudo. Se hicieron tres divisiones del interior al exterior de la pared del culmo en las rodajas (interna, media y externa). En la composición anatómica, la metodología fue similar a la caracterización de fibras hasta el corte de las rodajas del nudo y entrenudo. De estas se hicieron cortes histológicos transversales de $5 \mathrm{~mm}$ de espesor. La forma de los haces vasculares no coinciden completamente con la clasificación existente. El culmo está compuesto por $50 \%$ de parénquima, $41 \%$ de fibra y $9 \%$ de tejido conductivo. Las fibras miden en promedio 2,152 mm de longitud, 13,68 $\mu \mathrm{m}$ de diámetro, 3,29 $\mu \mathrm{m}$ de diámetro de lumen y 10,39 $\mu \mathrm{m}$ de grosor de pared.

PALABRAS CLAVE: caña brava, fibras de bambú, haces vasculares, parénquima, tarro, tejido conductivo.

\section{ABSTRACT}

The characterization of Guadua aculeata Rupr. ex Fournier culm wall was made; the specimens were collected on the locality "Las Margaritas", which is located in the municipality of Hueytamalco, Puebla, Mexico. The objective was to determine the proportion of the anatomical structures that make up the culm and the fiber sizes, to contribute to the species knowledge. The anatomical composition of the culm and the characterization of the fibers were studied, using four culms. For the measurement of the fiber, each culm was cut into three parts lengthwise (lower, middle and higher). From these section slices from the internode and close to the node, were cut. Three sections were obtained from the inside wall of the culm in the slices (inner, middle and outer). For the anatomical composition, the methodology was the same than that for fibers characterization, except for the cut slices of node and internode. From those, transverse histological sections $5 \mathrm{~mm}$ thick were made. The form of the vascular bundles does not coincide completely with the existing classification. The culm composition is $50 \%$ of parenchyma, $41 \%$ of fiber and $9 \%$ of conductive tissue. The fibers average dimension were 2,152 mm length, 13,68 $\mu \mathrm{m}$ diameter, 3,29 $\mu \mathrm{m}$ lumen diameter and 10,39 $\mu \mathrm{m}$ in wall thickness.

KEY WORDS: caña brava, bamboo fibers, vascular bundles, parenchyma cells, tarro, conductive tissue. 


\section{INTRODUCCIÓN}

El continente americano ocupa el segundo lugar en abundancia de especies de bambú, antecedido por Asia. De los países americanos, Brasil tiene la mayor cantidad de especies con alrededor de 140, seguido de Venezuela con 68 especies y Colombia 72, México se encuentra en el sexto lugar con 39. A lo largo del continente americano los géneros dominantes son: Elytrostachys, Arthrostylidium y Guadua. Las 26 especies de este último género se distribuyen desde el centro de México hasta el norte de Argentina, de 0 a 2200 metros sobre el nivel del mar (Bystriakova et al., 2004; Londoño, 2006; Cedeño e Irigoyen, 2011).

Las cinco especies de Guadua existentes en México son las que alcanzan las mayores dimensiones de todos los bambúes que se desarrollan en este país. De las cinco especies, Guadua aculeata llega a medir $25 \mathrm{~m}$ de altura y $25 \mathrm{~cm}$ de diámetro en la base. Es una especie que desarrolla espinas en todas las ramas, tiene entrenudos huecos de $20 \mathrm{~cm}$ a $30 \mathrm{~cm}$ de largo y de $2 \mathrm{~cm}$ de espesor (Cedeño e Irigoyen, 2011; Cortés, 2000).

Esta especie conocida como "tarro" es utilizada en la construcción de viviendas, principalmente, también en la elaboración de muebles, como cerca viva o sombra, entre otros. Los culmos son obtenidos por lo general de bambusales naturales dado que los cultivados son escasos. Si bien, en la actualidad se mencionan plantaciones de tarro, estas solo se encuentran en algunos estados como Puebla y Veracruz, sin estar documentada la superficie precisa (Ordóñez, 1999; Cortés, 2000; Cedeño e Irigoyen, 2011).

Como todas las gramíneas, la especie de interés está conformada por el rizoma, tallo o culmo, ramas, hojas, flores y frutos (Qisheng et al., 2003; Yang y Hui, 2010). El culmo es la parte del bambú más utilizada en la industria forestal (construcción, muebles, artesanías, entre otros), sin embargo la información tecnológica de la especie es poco documentada.

En general, el culmo está constituido por corteza (cutícula), haces vasculares y células de parénquima. Las proporciones y dimensiones de cada componente varían: entre especies, dentro de especies y dentro de un mismo individuo. Esto influye en la heterogeneidad de su densi- dad básica, contracciones, contenido de humedad, entre otras propiedades físicas, mecánicas y químicas (Liese, 1998; Londoño et al., 2002).

El conocimiento de las estructuras anatómicas de los culmos permitirá una mejor comprensión de su comportamiento en los procesos industriales a los cuales debe ser sometido para llegar a un producto final. Por lo tanto, conocer las características anatómicas se traduce en el adecuado manejo del material y a su vez en la optimización de los recursos.

\section{OBJETIVOS}

Conocer la proporción de las estructuras anatómicas que componen el culmo y las dimensiones de las fibras de Guadua aculeata, como una primera aportación al conocimiento de la especie.

\section{MATERIALES Y MÉTODOS}

La colecta del material se realizó en el predio "Las Margaritas" ubicado en el municipio de Hueytamalco, Puebla, México, a una altitud de 430 metros sobre el nivel del mar, entre las coordenadas geográficas $19^{\circ} 52^{\prime}$ y $20^{\circ}$ $12^{\prime}$ de latitud norte y $97^{\circ} 12^{\prime}$ y $97^{\circ} 23^{\prime}$ de longitud oeste. El predio se ubica sobre la carretera Hueytamalco-Ayotoxco de Guerrero, Puebla, aproximadamente a $12 \mathrm{~km}$ de la cabecera municipal de Hueytamalco, pasando la desviación a San José Acateno, Pue. (INEGI, 2009; INEGI, 2013).

La región presenta un clima cálido húmedo con abundantes lluvias en verano. Esta es una zona de transición con áreas reducidas de selva alta perennifolia; con vegetación secundaria arbustiva y bosque mesófilo de montaña. El material se recolectó en un bambusal natural (INEGI, 2009; INEGI, 2013).

Se utilizaron cuatro culmos de bambú de 9,5 m; 11,5 $\mathrm{m} ; 13,0 \mathrm{~m}$ y 13,6 m de longitud, de $5 \mathrm{~cm}, 6 \mathrm{~cm}, 7 \mathrm{~cm}$ y 8 $\mathrm{cm}$ de diámetro, respectivamente; libres de daños físicos, ataque de plagas y enfermedades. Cada uno de los culmos se segmentó en tres partes iguales (inferior, media y superior). Se cortó una rodaja de cada sección del entrenudo y cercana al nudo (Fig. 2). 


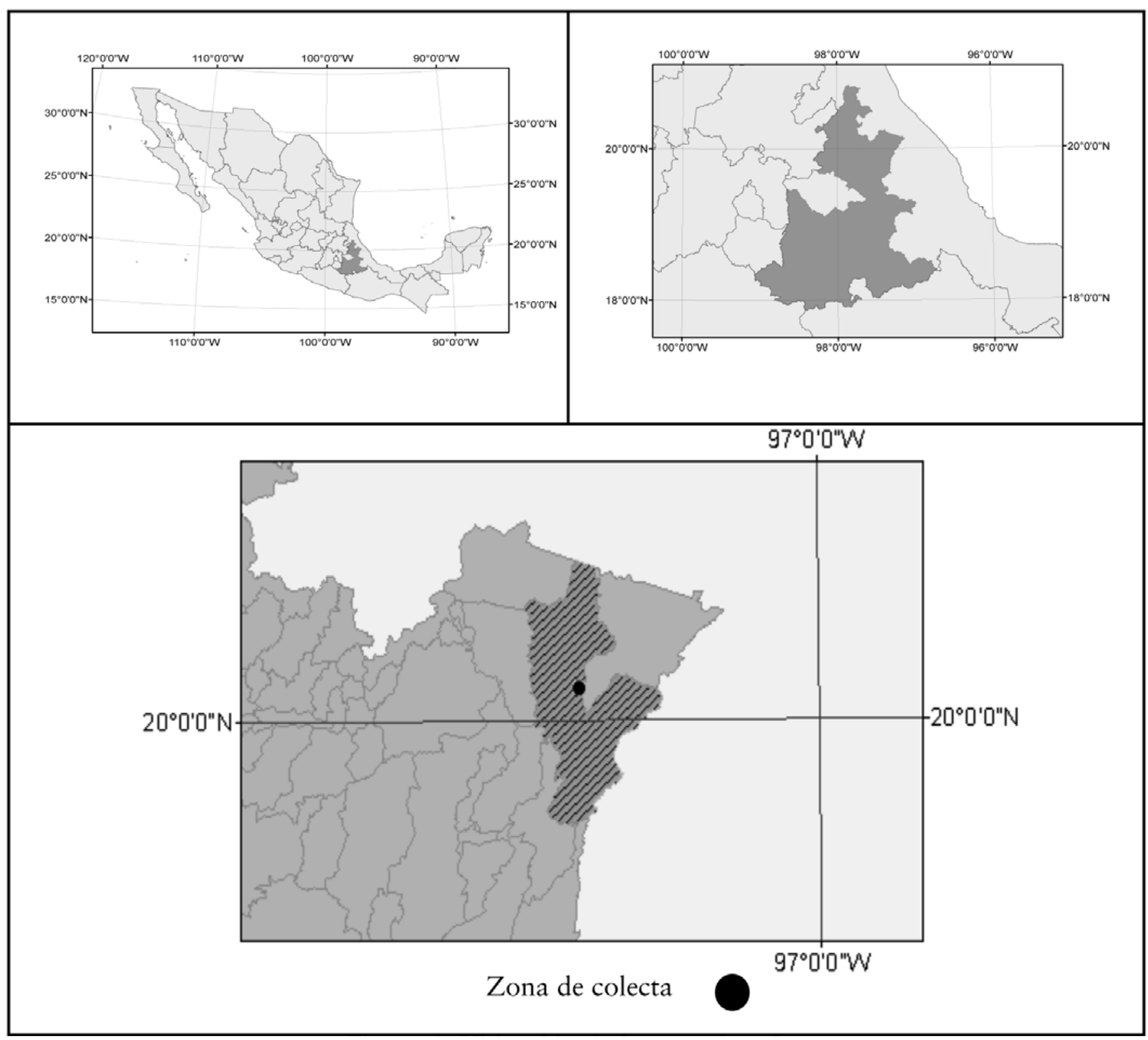

FIGURA 1. Ubicación de la zona de colecta.

La mitad de cada rodaja se colocó en un digestor diseñado y fabricado con las características determinadas por profesores-investigadores de la División de Ciencias Forestales de la Universidad Autónoma de Chapingo. Las muestras fueron sometidas a una presión de $2 \mathrm{~kg} / \mathrm{cm}^{2}$ durante dos minutos para ablandar las probetas. En seguida, de forma manual utilizando una sierra sable, se hicieron cortes transversales de $5 \mathrm{~mm}$ de espesor. En estas secciones se midieron el área que ocupan las fibras, parénquima y tejido conductivo (vasos), en una franja de un milímetro de ancho, desde el interior al exterior del culmo. Las áreas se determinaron con fotografías de resolución de 3,15 megapíxeles utilizando el software Leica Application Suite versión 3.7.0.

Para determinar el tamaño de muestra de la proporción de los elementos anatómicos constituyentes del 

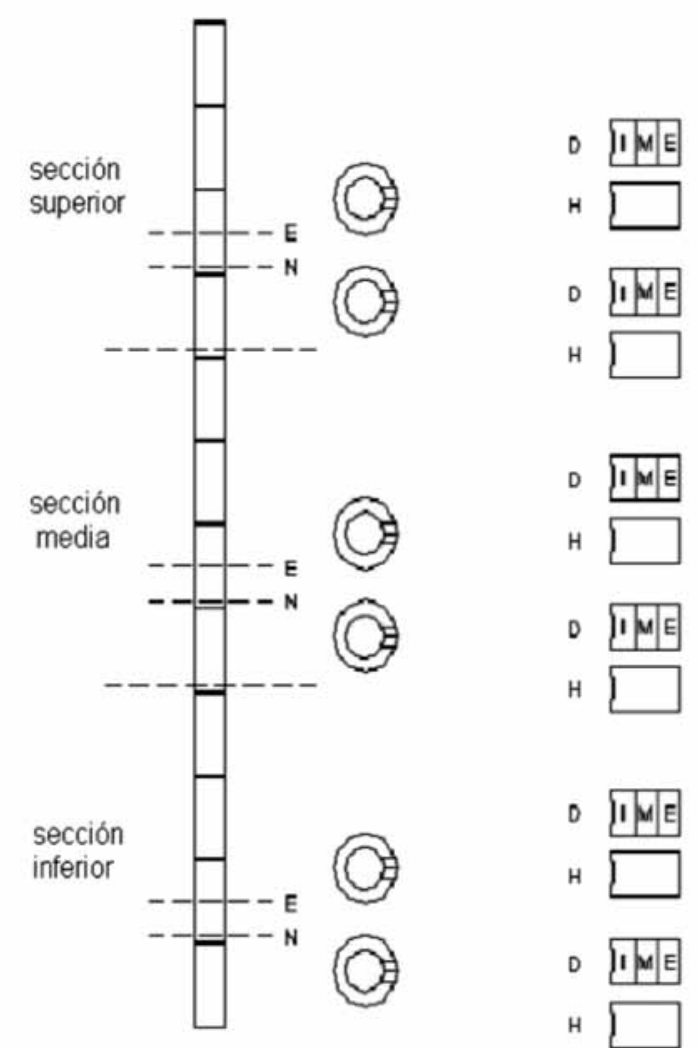
E: Sección del entrenudo
N. Sección cercana al nudo

D $1 \mathrm{ME}$

H

H. Cortes histológicos

$D$ M 1 E

H

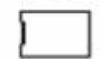

D IME

E: Sección externa

H

D $1 \mathrm{ME}$

H

D. Disociado

I: Sección interna

M. Sección media

FIGURA 2. Diagrama de la obtención de probetas para la descripción anatómica.

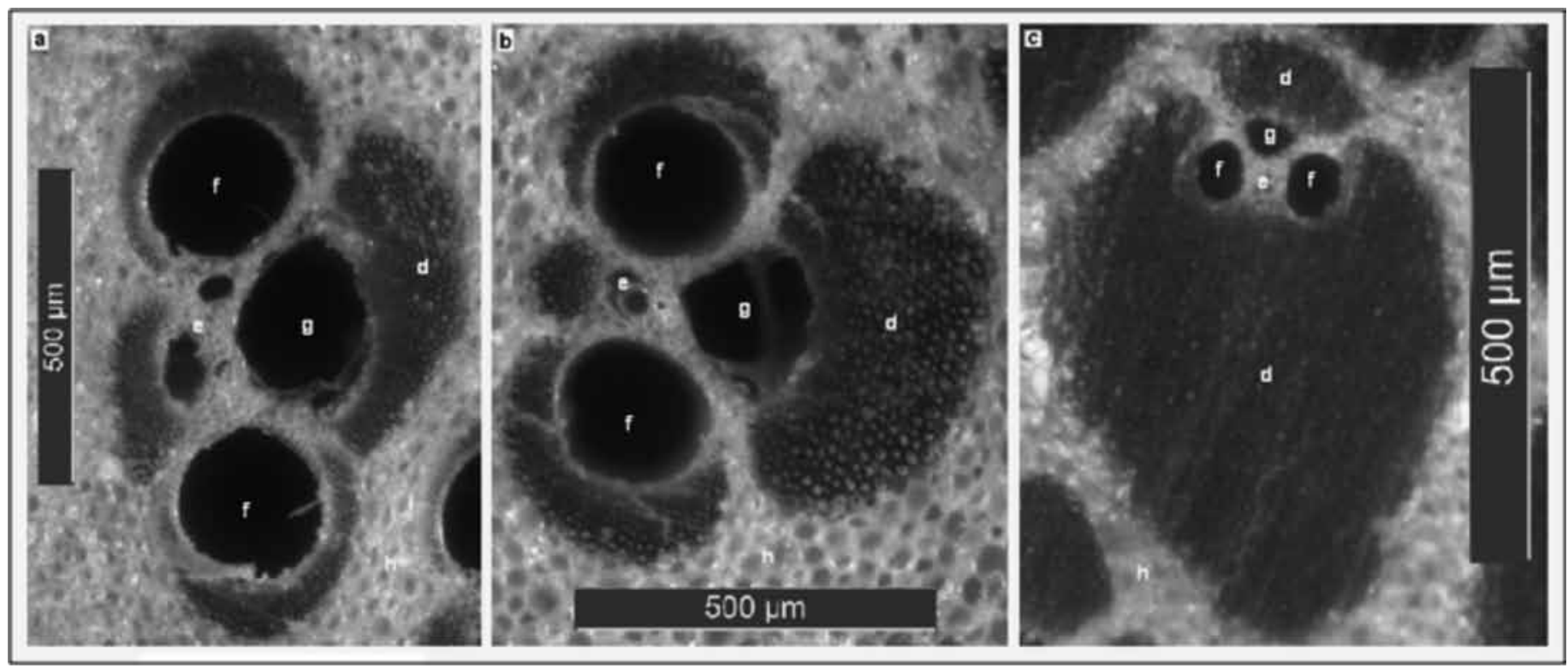

Figura 3. Haces vasculares de la sección interior (a), media (b) y exterior (c) del culmo; los tejidos que lo componen son las fibras (d), protoxilema (e), el metaxilema (f), el floema (g) y el parénquima (h). 
culmo, se consideró un nivel de confianza de $10 \%$, obteniendo un resultado de 12 franjas a medir. Se utilizó la siguiente fórmula (Gilbert, 1987):

Donde:

$$
n=\frac{\left(t^{2} \times c^{2}\right)}{d^{2}}
$$

$n$ : Tamaño de muestra.

$t$ : Valor de $t$ para la confiabilidad deseada.

$c$ : Coeficiente de variación.

$d$ : nivel de confianza

De la otra mitad de la rodaja se obtuvieron astillas para la preparación de material macerado. Las astillas se colocaron en frascos con solución de ácido acético y peróxido de hidrógeno 50/50. Este material se colocó en una estufa a una temperatura de $60^{\circ} \mathrm{C}$ durante 60 horas con el fin de eliminar la lámina media de los elementos anatómicos.

El macerado se lavó con agua destilada, se tiñó con pardo de Bismark y se elaboraron preparaciones temporales. Se midieron la longitud y los diámetros de las fibras, y los lúmenes con una cámara Leicaicc50 con una resolución de 3 megapíxeles y el software Leica Application Suite versión 1.8. El número de fibras por medirse calculó aplicando la misma fórmula utilizada en los elementos anatómicos constituyentes del culmo y un nivel de confianza de $5 \%$, obteniendo un tamaño de muestra de 55 fibras para cada una de la secciones del culmo.

Los índices de calidad de pulpa de papel se calcularon utilizando los valores medios de longitud, grosor de paredes de la fibra, diámetro de lúmenes y diámetro de las fibras.

Coeficiente o índice de rigidez: C.R. $=2 w / D$
Coeficiente de flexibilidad: C.F. $=l / D$

Coeficiente de Peteri o índice de esbeltez: I.E. $=L / D$

Relación de Runkel: R.R. $=2 w / l$

Donde:

D: diámetro de la fibra

$L$ : longitud de la fibra

l: diámetro del lumen

$2 w$ : grosor de la pared de la fibra

\section{Resultados}

Los haces vasculares observados en G. aculeata están formados por dos vasos de metaxilema, uno o más vasos de floema, uno o más vasos de protoxilema y un conjunto de fibras que rodean a los elementos conductivos. Los haces cercanos al interior del culmo (Fig. 3a) presentan un grupo de fibras rodeando en mayor proporción a los vasos de metaxilema y principalmente al floema, y un grupo menor rodea al protoxilema, esta distribución de los elementos anatómicos se modifica del interior al exterior del culmo.

En la sección media de la pared del culmo, el conjunto de fibras que rodea al protoxilema aumenta, en algunos casos, hasta igualarse al grupo de fibras que rodea al floema (Fig. 3b). Conforme se acerca al exterior del culmo, la mayor proporción de fibras rodea al protoxilema, sin dejar de cubrir los alrededores del floema (Fig. 3c).

La proporción de los elementos constituyentes de la pared del culmo en dirección axial (inferior, media y superior) de secciones del entrenudo y cercanas al nudo se presentan en la tabla 1. En la tabla 2 se presentan las medidas de fibras de la sección interna, media y externa, del nudo y entrenudo, de la zona inferior, media y superior del culmo.

TABLA 1. Composición de la pared del culmo en dirección axial.

\begin{tabular}{llllllll}
\hline \multicolumn{1}{c}{ Estructura (\%) } & $\begin{array}{c}\text { Entrenudo } \\
\text { inferior }\end{array}$ & $\begin{array}{c}\text { Nudo } \\
\text { inferior }\end{array}$ & $\begin{array}{c}\text { Entrenudo } \\
\text { medio }\end{array}$ & $\begin{array}{c}\text { Nudo } \\
\text { medio }\end{array}$ & $\begin{array}{c}\text { Entrenudo } \\
\text { superior }\end{array}$ & $\begin{array}{c}\text { Nudo } \\
\text { superior }\end{array}$ & Promedio \\
\hline Fibra (\%) & $39 \mathrm{~A}$ & $36 \mathrm{~B}$ & $43 \mathrm{~A}$ & $44 \mathrm{~A}$ & $43 \mathrm{~A}$ & $42 \mathrm{~A}$ & 41 \\
Parénquima & $54 \mathrm{~A}$ & $57 \mathrm{~A}$ & $47 \mathrm{~B}$ & $47 \mathrm{~B}$ & $45 \mathrm{~B}$ & $49 \mathrm{~B}$ & 50 \\
Poros & 7D & $6 \mathrm{D}$ & $10 \mathrm{~B}$ & $10 \mathrm{~B}$ & $12 \mathrm{~A}$ & $9 \mathrm{C}$ & 9 \\
\hline
\end{tabular}


a las secciones cercanas al nudo. 
TABLA 2. Dimensiones de fibras en dirección axial y transversal.

\begin{tabular}{|c|c|c|c|c|c|c|c|c|}
\hline \multirow{2}{*}{$\begin{array}{l}\frac{0}{0} \\
\frac{\pi}{\frac{\pi}{\pi}} \\
>\end{array}$} & \multirow{2}{*}{$\begin{array}{l}\text { Sección } \\
\text { Iongitud }\end{array}$} & \multicolumn{3}{|c|}{ Sección entrenudo } & \multicolumn{3}{|c|}{ Sección cercana al nudo } & \multirow{2}{*}{ Promedic } \\
\hline & & Interno & Medio & Externo & Interno & Medio & Externo & \\
\hline \multirow{7}{*}{  } & \multirow{2}{*}{ Inferior } & 2,15 & 2,66 & 2,56 & 2,22 & 2,44 & 2,52 & 2,42 \\
\hline & & B & $A$ & A & B & A & A & \\
\hline & \multirow{2}{*}{ Media } & 1,84 & 2,25 & 2,13 & 1,93 & 2,15 & 2,13 & 2,07 \\
\hline & & C & B & B & C & B & B & \\
\hline & \multirow{2}{*}{ Superior } & 1,84 & 2,22 & 2,12 & 1,66 & 1,99 & 1,96 & 1,96 \\
\hline & & C & $B$ & B & C & B & B & \\
\hline & Promedio & 1,94 & 2,38 & 2,27 & 1,93 & 2,19 & 2,20 & 2,15 \\
\hline \multirow{7}{*}{  } & \multirow{2}{*}{ Inferior } & 13,58 & 16,72 & 15,12 & 13,29 & 15,68 & 14,11 & 14,75 \\
\hline & & B & $A$ & A & B & A & B & \\
\hline & \multirow{2}{*}{ Media } & 12,33 & 14,14 & 14,06 & 12,69 & 14,37 & 14,47 & 13,68 \\
\hline & & C & B & B & B & B & B & \\
\hline & \multirow{2}{*}{ Superior } & 12,22 & 13,42 & 12,62 & 12,15 & 13,02 & 12,21 & 12,61 \\
\hline & & C & B & B & C & B & C & \\
\hline & Promedio & 12,71 & 14,76 & 13,93 & 12,71 & 14,36 & 13,60 & 13,68 \\
\hline \multirow{7}{*}{  } & \multirow{2}{*}{ Inferior } & 3,29 & 3,92 & 3,36 & 3,25 & 3,64 & 3,52 & 3,50 \\
\hline & & $A$ & $A$ & A & A & A & A & \\
\hline & \multirow{2}{*}{ Media } & 3,03 & 3,29 & 3,79 & 3,10 & 3,65 & 3,36 & 3,37 \\
\hline & & B & $A$ & A & A & A & A & \\
\hline & \multirow{2}{*}{ Superior } & 3,07 & 3,26 & 3,04 & 2,69 & 3,01 & 2,99 & 3,01 \\
\hline & & B & $A$ & B & B & B & B & \\
\hline & Promedio & 3,13 & 3,49 & 3,40 & 3,01 & 3,44 & 3,29 & 3,29 \\
\hline \multirow{7}{*}{ 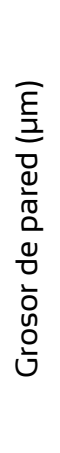 } & \multirow{3}{*}{ Inferior } & 5,15 & 6,40 & 5,88 & 5,02 & 6,02 & 5,29 & 5,63 \\
\hline & & B & $A$ & A & B & A & B & \\
\hline & & 4,65 & 5,43 & 5,13 & 4,80 & 5,36 & 5,55 & 5,15 \\
\hline & Media & C & B & B & B & B & B & \\
\hline & \multirow{2}{*}{ Superior } & 4,58 & 5,08 & 4,79 & 4,73 & 5,00 & 4,61 & 4,80 \\
\hline & & C & B & B & B & B & C & \\
\hline & Promedio & 4,79 & 5,64 & 5,27 & 4,85 & 5,46 & 5,15 & 5,19 \\
\hline
\end{tabular}

Los promedios con letras iguales presentan diferencia no significativa entre ellas con un nivel de significancia de $5 \%$. 
Con las dimensiones obtenidas en este trabajo se calcularon el coeficiente de rigidez igual a 0,76 ; el coeficiente de flexibilidad igual a 0,24; el índice de esbeltez igual 162,69 y la relación de Runkel igual a 3,29.

\section{DISCUSIÓN}

La forma de los haces vasculares es semejante, en la periferia, a los de Guadua trinii y Guadua chacoensis. En la sección interna de G. aculeata se encontraron haces similares en forma a los de G. chacoensis, G. trinii y Guadua paraguayensis (Rúgolo de Agrasar y Rodríguez, 2003). Se confirmó, además, la variación de la forma, tamaño y distribución en las direcciones trasversal y longitudinal de los haces, señalado por Liese (1998).

Las características de forma y distribución de los componentes de los haces vasculares no coincidió completamente con ninguno de los tipos de haces descritos por Liese (1998). Sin embargo, presentó características del tipo II y IIa, como la mayor cantidad de fibras que rodea al floema comparada con los costados del haz y, conforme se acerca a la periferia, el conjunto de fibras se hace mayor hacia al lado del protoxilema (Liese, 1998).

La proporción menor de fibras se ubicó en la zona cercana al nudo inferior, siendo la única que presentó diferencias significativas con el resto de las zonas. El promedio de la proporción de fibras obtenido para el material en estudio, fue semejante a los valores de Guadua angustifolia que contiene $40 \%$, valor señalado como generalidad en los bambúes (Liese, 1998; Londoño et al., 2002). En la zona interna del culmo, los haces vasculares resultaron mayores que los de la zona externa, asimismo se identificó que los espacios vacíos (protoxilema, metaxilema y floema) tenían mayor dimensión, que los haces se encuentran más separados entre sí, y que la proporción de parénquima incrementa en comparación con el último tercio hacia el exterior de culmo.

El parénquima del material estudiado, presentó la mayor proporción de las estructuras anatómica de los culmos; el promedio obtenido para esta especie fue cercano a $52 \%$, valor promedio para los bambúes en general, y similar a los de G. angustifolia obtenidos por Liese (1998) y
Londoño et al. (2002) que obtuvieron 51\%. En la parte inferior de los culmos se identificaron las mayores cantidades de parénquima, siendo estadísticamente diferente a los resultados obtenidos para las secciones media y superior.

El tejido conductivo presentó el menor porcentaje de elementos en la composición del culmo, siendo semejante al obtenido para G. angustifolia de 9\% según Londoño (2002) y similar al promedio generalizado de $8 \%$ para los bambúes, señalado por Liese (1998). En este estudio se encontró que el entrenudo superior contiene las proporciones mayores de este tejido, resultando significativamente diferente de los valores registrados para las zonas inferior, media y cercana al nudo superior del culmo.

Las longitudes de la fibras obtenidas para G. aculeata variaron entre $1,66 \mathrm{~mm}$ y $2,66 \mathrm{~mm}$, valores mayores a los encontrados por Omobowale y Ogedengbe (2008) en Bambusa vulgaris $(1,40 \mathrm{~mm}-1,80 \mathrm{~mm})$ y en Gingantchloa levis (1,82 mm) estudiada por Espiloy (1985). Sin embargo, son menores a los de Bambusa blumeana (2,56 $\mathrm{mm})$, B. vulgaris $(2,37 \mu \mathrm{m}-3,70 \mu \mathrm{m})$ y G. levis $(3,76 \mathrm{~mm}$ - 4,48 mm) (Espiloy, 1985; Ogunsile y Uwajeh, 2009; Wahab et al., 2009; Guimarães et al., 2010; Nordahlia et al., 2012).

Del interior al exterior del culmo, las fibras que presentaron mayor longitud del entrenudo y cercanas al nudo se ubican en el centro y periferia de la pared del culmo, las de menor largo en el interior (Tabla 2). De acuerdo con las observaciones realizadas en cortes histológicos y las mediciones en otras especies, esto puede deberse al proceso de envejecimiento de la pared del culmo, que se observa más marcado en la zona interna del culmo. Esta condición se traduce en una menor densidad en la parte interna del culmo en comparación con la periferia, lo cual se debe tomar en cuenta para el uso del material (Liese y Weiner, 1996).

La longitud promedio de las fibras obtenida disminuyó de la base hacia el ápice del culmo; tendencia similar a lo publicado por Omobowale y Ogedengbe (2008) para Bambusa vulgaris, Wahab et al. (2009) para B. vulgaris, y Nordahlia et al. (2012) para Gingantchloa levis, esto puede deberse a que en la parte superior del culmo se 
encuentran las células más jóvenes, es decir, no han alcanzado la madurez, condición diferente a las estructuras de las parte inferior.

El diámetro de la fibra aquí encontrado varió de $12,15 \mu \mathrm{m}$ a $16,72 \mu \mathrm{m}$ (Tabla 2), estos valores tienen similitud con B. blumeana $(14,2 \mu \mathrm{m}-15,2 \mu \mathrm{m})$ y G. levis $(15,5 \mu \mathrm{m}-16,5 \mu \mathrm{m})$. El promedio de esta variable en el culmo de G. aculeata resultó menor al publicado para $G$. levis $(16,0 \mu \mathrm{m}-25,7 \mu \mathrm{m})$, B. vulgaris $(13,93 \mu \mathrm{m}-27,3$ $\mu \mathrm{m})$ (Espiloy, 1985; Ogunsile y Uwajeh, 2009; Wahab et al., 2009; Guimarães et al., 2010; Nordahlia et al., 2012).

Las dimensiones de los diámetros de la fibra de las secciones media y externa de la pared del culmo del entrenudo fueron mayores que las de la interna, siendo estadísticamente diferentes. En las secciones cercanas al nudo, las zonas inferior y superior presentaron la mayor longitud en parte media de la pared, existiendo diferencias significativas con las secciones internas y externas; para la zona media del largo del culmo no se registraron estas diferencias. El diámetro promedio de la fibra disminuyó de la base hacia el ápice, esta tendencia coincide con los resultados de Espiloy (1985) para B. blumeana y los de Wahab et al. (2009) para B. vulgaris. Al igual que para la longitud de fibra, esta situación se puede deber a que las estructuras más jóvenes se ubican en la parte superior del culmo.

El promedio general del diámetro del lumen que se obtuvo fue de 3,29 $\mu \mathrm{m}$, el valor mínimo fue de 2,69 $\mu \mathrm{m}$ y el máximo de 3,92 $\mu \mathrm{m}$, los valores se aproximan a lo publicado para B. blumeana $(3,2 \mu \mathrm{m}-4,6 \mu \mathrm{m})$ y $B$. vulgaris $(2,4 \mu \mathrm{m}-2,6 \mu \mathrm{m})$. La media del culmo es menor a lo registrado para G. levis $(5 \mu \mathrm{m}-16 \mu \mathrm{m})$ y B. vulgaris $(8 \mu \mathrm{m}$ - 10,4 нm) (Espiloy, 1985; Sekyere, 1994; Ogunsile y Uwajeh, 2009; Wahab et al., 2009; Guimarães et al., 2010; Nordahlia et al., 2012). El diámetro de lumen disminuyó de la base a la parte superior del culmo, con diferencias significativas entre la parte superior cercana al nudo, las secciones superiores externa e interna del entrenudo y la sección interna a la altura media del culmo con el resto de las secciones.
El grosor de pared de la fibra que se midió fue de 4,58 $\mu \mathrm{m}$ a $6,40 \mu \mathrm{m}$, similar al que se ha publicado para B. blumeana $(5,2 \mu \mathrm{m}-6,1 \mu \mathrm{m})$ y $B$. vulgaris $(3,29 \mu \mathrm{m}-7,10 \mu \mathrm{m})$ y menor al de G. levis (10 $\mu \mathrm{m}-12 \mu \mathrm{m})$. En el entrenudo, se obtuvieron diferencias significativas entre las secciones exterior y media de la pared del culmo con la interna; en las secciones cercanas al nudo, estas diferencias se presentaron entre la sección interna con ambos lados en la parte inferior y entre la exterior con las media e interna de la parte superior (Espiloy, 1985; Sekyere, 1994; Ogunsile y Uwajeh, 2009; Wahab et al., 2009; Guimarães et al., 2010; Nordahlia et al., 2012).

El grosor de la pared influye directamente en la densidad básica, propiedad que se ve reflejada en otras propiedades físicas y mecánicas. Por otro lado, las fibras de bambú tienen estructura polilaminar; en la madurez se presentan la mayor cantidad de láminas, característica observada en G. aculeata. Las fibras son las estructuras de soporte, entre mayor grosor presentan aumenta la resistencia mecánica; las paredes gruesas de G. aculeata junto con la estructura polilaminar hacen de sus culmos adecuados para su uso en la construcción (Liese, 1998).

De acuerdo con las clasificaciones que existen los valores obtenidos para los coeficientes de rigidez y de flexibilidad, el índice de esbeltez y la relación de Runkel indican que las fibras de G. aculeata son largas pero con paredes gruesas, por lo tanto, es un material de mala calidad para la elaboración de papel (Porres y Valladares, 1979; Tortorelli, 1956).

\section{CONCLUSIONES}

Este trabajo significa una de las primeras aportaciones para el estudio de la anatomía de las paredes de los tallos de bambúes nativos de México.

Las estructuras anatómicas identificadas y medidas, así como las proporciones y distribución dentro de las paredes de los tallos se catalogan dentro de los valores promedio que se han publicado para otras especies de bambú.

Por el tamaño y proporción de sus fibras, además del grueso de sus paredes puede considerarse como una especie adecuada para ser utilizada en la construcción. 
La relación entre la longitud y el grosor de la pared de las fibras indican que no es idónea para la elaboración de papel.

\section{REFERENCIAS}

Bystriakova, N., V. Kapos e I. Lysenko. 2004. Africa, Madagascar and the Americas. International Network for Bamboo and Rattan. Beijing. 90 p.

Cedeño V., A. y J. Irigoyen C. 2011. El bambú en México. USJT Arq. Urb. 6:223-243.

Cortés R., G.R. 2000. Los bambúes nativos de México. Conabio. Biodiversitas 30:12-15.

Espiloy, Z.B. 1985. Physico-mechanical properties and anatomical relationships of some Philippine bamboos. International Bamboo Workshop. China. p:257-264.

Gilbert, R.O. 1987. Statistical methods for environmental pollution monitoring. Van Nostrand Reinhold. 320 p.

Guimarães Junior, M., K. Monteiro-Novack y V.R. Botaro. 2010. Caracterização anatômica da fibra de bambu (Bambusa vulgaris) visando sua utilização em compósitos poliméricos. Revista Iberoamericana de Polímeros 11(7):442-456.

INEGI (Instituto Nacional de Estadística y Geografía). 2009. Prontuario de información geográfica municipal de los Estados Unidos Mexicanos. Disponibles en: de: http:// www3.inegi.org.mx/sistemas/mexicocifras/datos-geograficos/21. Consultada el 10/12/2013.

INEGI (Instituto Nacional de Estadística y Geografía). 2013. Principales tipos de vegetación de México. Disponible en: http://mapserver.inegi.gob.mx/geografia/espanol/ datosgeogra/vegfauna/vegeta.cfm. Consultada el 10/12/2013.

Liese, W. 1998. The anatomy of bamboo culms. INBAR Technical Report $\mathrm{N}^{\circ}$ 18. International Network for Bamboo and Rattan. Beijing. 204 p.

Liese, W. y G. Weiner. 1996. Ageing of bamboo culms. A review. Wood Science and Technology 30:77-89

Londoño, X. 2006. Aspectos generales de los bambúes americanos. Revista electrónica Bio bambú. Disponible en: http:// www.bambumex.org/ paginas/ASPECTOS\%20GENERALES. pdf. Consultada el 10/09/2011.
Londoño, X., G.C. Camayo, N.M. Riaño y Y. López. 2002. Characterization of the anatomy of Guadua angustifolia (Poaceae: Bambusoideae) culms. Bamboo Science and Culture: The Journal of the American Bamboo Society $16(1): 18-31$

Nordahlia, A.S., U.M.K. Anwar, H. Hamdan, A. Zaidon, M.T. Paridah y O. Abd Razak. 2012. Effects of age and height on selected properties of malasyan bamboo (Gigantochloa levis). Journal of Tropical Forest Science 24(1):102-109.

Ogunsile, B.O. y C.F. Uwajeh. 2009. Evaluation of the pulp and paper potentials of a Nigerian grown Bambusa vulgaris. World Applied Sciences Journal 6(4):536-531.

Omobowale, M.O. y K. Ogedengbe. 2008. Trends and fiber characteristics of Nigerian grown bamboo and its effect in its impact and tensile strengths. Bamboo Science and Culture: The Journal of the American Bamboo Society 21(1):9-13.

Ordóñez C., V.R. 1999. Perspectivas del bambú para la construcción en México. Madera y Bosques 5(1):3-12.

Porres, C. y J. Valladares. 1979. Producción de pulpa y papel con materias primas autóctonas centroamericanas. Instituto Centroamericano de Investigaciones y Tecnología Industrial. Guatemala. 72 p.

Qisheng, Z., J. Shenxue y T. Yongyu. 2003. Industrial utilization on bamboo. INBAR Technical Report $\mathrm{N}^{\circ} 26$. International Network for Bamboo and Rattan. Beijing. 207 p.

Rúgolo de Agrasar, Z.E. y M.F. Rodríguez. 2003. Culm anatomy of native woody bamboos in Argentina and neighboring areas: Cross section. Bamboo Science and Culture: The Journal of the American Bamboo Society 17(1): 28-43.

Sekyere, D. 1994. Potencial of bamboo (Bambusa vulgaris) as a source of raw material for pulp and paper in Ghana. Ghana Journal of Forestry 1:49-56

Tortorelli L., A. 1956. Maderas y bosques argentinos. ACME. Buenos Aires. 910 p.

Wahab, R., A. Mohamed, M.T. Mustafa y A. Hassan. 2009. Physical characteristics and anatomical properties of cul- 
tivated bamboo (Bambusa vulgaris Schrad) culms. Journal of Biological Sciences 9(7):753-759.

Yang, Y. y C. Hui. 2010. China's bamboo. INBAR Technical Report $\mathrm{N}^{\circ}$ 33. International Network for Bamboo and Rattan. Beijing. 227 p.
Manuscrito recibido el 29 de junio de 2012

Aceptado el 2 de mayo de 2014.

Este documento debe citarse como:

Zaragoza-Hernández, I., A. Borja de la Rosa, F.J. Zamudio-Sánchez, V.R. Ordóñez-Candelaria y G.M. Bárcenas-Pazos. 2014. Anatomía del culmo de bambú (Guadua aculeata Rupr.) de la región nororiental del estado de Puebla. Madera y Bosques 20(3):87-96. 